\title{
Tissue ablation with 100-fs and 200-ps laser pulses
}

\author{
N. Nishimura, C. B. Schaffer, E. H. Li ${ }^{\dagger}$, and E. Mazur* \\ Harvard University, Department of Physics, Cambridge, MA 02138
}

\begin{abstract}
We used water and human skin tissue to compare the surgical potential of 100-fs and 200-ps laser pulses. For investigation of threshold behavior of 100-fs and 200-ps pulses, we use water as a model for tissue. In addition to having a lower threshold, we find that energy deposition is much more consistent with 100 -fs pulses. We also compared 100-fs and 200-ps laser pulse effects on the surface and in the bulk of human skin tissue. On the surface, pulses with 100-fs and 200-ps duration leave similar size ablation regions. In the bulk both 100 -fs and 200 -ps pulses produce cavities, however, 100 -fs pulses result in a smaller cavity size. On both the surface and in the bulk 100 -fs pulses show less collateral tissue damage than 200 -ps pulses.
\end{abstract}

Keywords: femtosecond lasers, tissue ablation, laser surgery, laser induced breakdown

${ }^{\dagger}$ Current address: Department of Electrical and Electronic Engineering, University of Hong Kong

*E-mail: mazur@physics.harvard.edu WWW: http://mazur-www.harvard.edu

\section{Introduction}

Ultrashort pulses may provide an ideal mechanism for surgery, especially in transparent (or nearly transparent) tissues, i.e., tissues that do not normally absorb the laser radiation. When a femtosecond laser pulse is tightly focused inside a transparent material, the intensity in the focal volume can become high enough to cause absorption through nonlinear processes.[1] Because the absorption is nonlinear, it takes place at the focus inside the material instead of at the surface. The absorption produces a micrometer-sized, highly-excited plasma trapped inside the material. This plasma expands into the surrounding volume, creating a microscopic explosion - a microexplosion.[2] In tissue, the microexplosion can be used to precisely vaporize tissue while minimizing damage to surrounding tissue.

Laser-induced tissue ablation is a multi-stage process. First, tissue is ablated by the hot plasma formed at the focal volume of the laser. Second, the plasma expands with supersonic velocity, and drives a shock wave.[3] As the shock wave propagates outward, it decays into an acoustic pressure wave[4]; the region of supersonic expansion is called the shock zone. Next, a cavitation bubble is formed by the expansion of vaporized material. The bubble expands outward from the focal region until, after tens of microseconds, it collapses under external pressure. The laser-produced plasma vaporizes tissue, providing the surgical effect. However, both the shock wave and the cavitation bubble propagation create a surrounding region of partial damage due to mechanical disruption. [5], [6]

Most clinical applications employ 10-ns pulses from a $\mathrm{Nd}$ :YAG laser with pulse energies greater than $1 \mathrm{~mJ}$. These pulses produce large shock zones which limit the surgical precision and cause collateral tissue damage.[7],[8] Furthermore, unwanted energy deposition can occur in any absorbing tissue outside the focal region.[7]

Several recent experiments [8]-[12] have shown that picosecond and femtosecond lasers may allow for smaller ablation regions as well as more limited shock zones and cavitation bubble sizes. Using a time-resolved imaging technique discussed elsewhere [13] we find that the shock zone radius in water created by $10-\mu \mathrm{J}, 100$-fs pulses is only 8.5 $\mu \mathrm{m}$. In contrast, other researchers [4] have found that with picosecond pulses at the same laser energy the shock zone radius is $50 \mu \mathrm{m}$. Furthermore, with $1-\mathrm{mJ}$ nanosecond pulses in saline solution the explosion front propagates at supersonic speeds for nearly $100 \mu \mathrm{m}$.[14] The cavitation bubble reaches a radius of $\sim 500 \mu \mathrm{m}$.[14] The increased size of mechanical effects is due to the higher threshold for breakdown with longer pulses. Longer pulses require more energy to initiate breakdown, and thus when breakdown occurs they deposit more energy into the sample, leading to larger shock zones and cavitation bubbles.

In this paper, we report on femtosecond and picosecond laser-induced breakdown in water and human skin tissue. In water, we find a much lower breakdown threshold for femtosecond pulses, with more consistent energy deposition near the threshold. In skin, we investigate surface ablation and sub-surface cavity formation with both femtosecond and picosecond pulses. We find that similar size surface ablation is produced with both pulse lengths, but that the shorter pulses produce smaller sub-surface cavities. Furthermore, for both surface ablation and sub-surface cavity formation, the extent of collateral damage is greatly reduced by using femtosecond instead of picosecond laser pulses. 


\section{Threshold behavior in water}

In water, breakdown thresholds were determined by measuring the laser energy required to launch a pressure wave which was detected acoustically. We performed measurements using 100-fs and 200-ps, 800-nm pulses from an amplified Ti:Sapphire laser. A 0.65 numerical aperture (NA) objective is used to focus the pulses inside a water cell containing a submerged piezoelectric sensor (see inset to Fig.1). We amplify the piezoelectric voltage and record the signal on a digital oscilloscope. The amplitude of the response increases with increasing energy, but the shape of the response changes only slightly. Using the height of the first peak, we measure the magnitude of the pressure in the wave as a function of input energy. Figure 1 shows, on a logarithmic scale, the pressure produced by $100-\mathrm{fs}$ and 200 -ps pulses over three orders of magnitude in laser pulse energy. Above $10 \mu \mathrm{J}$, the pressure produced by the $100-\mathrm{fs}$ and 200 -ps pulses is equal. In the range $10-100 \mu \mathrm{J}$ the logarithmic slope is 0.5 , indicating that the pressure increases approximately as the square-root of the input energy and that the deposited energy is proportional to the input energy.

With 200-ps pulses no clear breakdown threshold exists. In the range $2-6 \mu \mathrm{J}$, as the energy increases, an increasing percentage of the laser shots produce breakdown, with no breakdown below $2 \mu \mathrm{J}$ and breakdown on every shot above 6 $\mu \mathrm{J}$. In contrast, with 100 -fs pulses there is little shot-to-shot variation in the pressure produced by the breakdown even very close to the threshold. Below $1 \mu \mathrm{J}$, the absorbed fraction of each pulse diminishes with decreasing incident energy, but the breakdown occurs consistently from shot-to-shot. This difference in behavior occurs because the energy deposition for 100 -fs pulses is initiated by multiphoton absorption, whereas the absorption of 200-ps pulses relies on absorbing impurities to generate the initial electrons.

For pulses with energies greater than $10 \mu \mathrm{J}$ we find a transmission of $20 \%$ through the water sample, indicating that a large fraction of the incident energy is absorbed. Assuming $80 \%$ absorption in this energy range, we can use the pressure measurements to extrapolate the absorption coefficient to smaller energies. The low pressure produced by $100-\mathrm{fs}$ pulses near the $0.2-\mu \mathrm{J}$ threshold indicates that it is possible to consistently deposit a small fraction of the incident energy into the pressure wave - approximately $50 \mathrm{pJ}$.

\section{Tissue ablation and sub-surface cavity forma- tion}

We examined the differences between femto- and picosecond laser-induced breakdown in tissue. Pulsed lasers are of medical interest for their precision and their ability to cut beneath a surface without damaging the layers above the focus.[7] We compared the effects of single 100-fs and 200ps Ti:Sapphire laser pulses on the surface and in the bulk of skin. We used an in vitro, stratified human skin model, Epi-

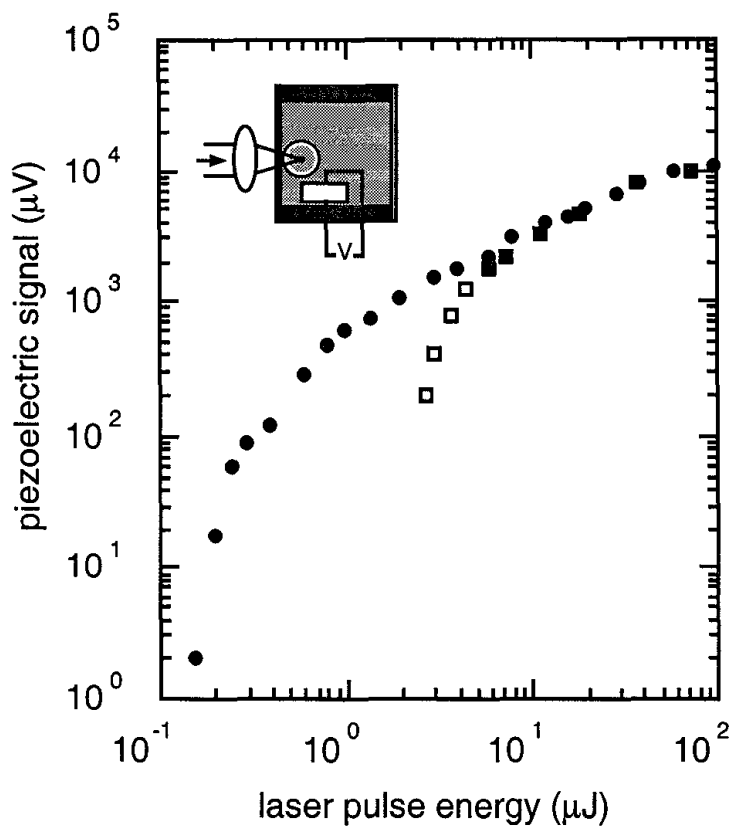

Fig. 1. Comparison of the piezoelectric signal as a measure of the pressure produced by 100 -fs pulses (filled circles) and 200 -ps pulses (squares) in water. Each point is averaged over 100 pulses. For the 200-ps data, the open squares represent a range in pulse energy where some pulses produce no signal, while others significantly exceed the average. Inset shows the water cell for piezoelectric pressure measurements.

Derm $^{\mathrm{TM}}$, from MatTek Corporation. The 100-fs and 200-ps pulses were focused with a 0.65 NA microscope objective. The samples were translated during irradiation so that each laser pulse left a single isolated damaged region. For crosssectional views, the samples were stained in toluidine blue, fixed in $10 \%$ formalin, then sliced in $30-\mu \mathrm{m}$ sections using a vibratome and photographed with an optical microscope. For top-views, photographs of unstained samples were taken with an optical microscope.

We compared laser ablation on the skin surface using 100 -fs and 200-ps pulses. We find that although ablation spot size is similar for femtosecond and picosecond pulse lengths at similar laser energies, surrounding tissue damage is much greater for picosecond pulses. Figure $2 a$ and $2 b$ show topview photographs of skin surface ablated by 200 -ps and $100-$ fs pulses at $40-\mu \mathrm{J}$ energy. The 100 -fs pulse ablated region has a diameter of $\sim 14 \mu \mathrm{m}$, while the 200-ps pulse ablated region is about $\sim 12 \mu \mathrm{m}$ in diameter. However, the picosecond ablation diameter is difficult to define because the edge of the ablated region is jagged. The picosecond ablated region is surrounded by damaged tissue indicated by darkened, uneven areas and white, shiny areas with diameters up to $50 \mu \mathrm{m}$. By contrast, the femtosecond ablated region has 


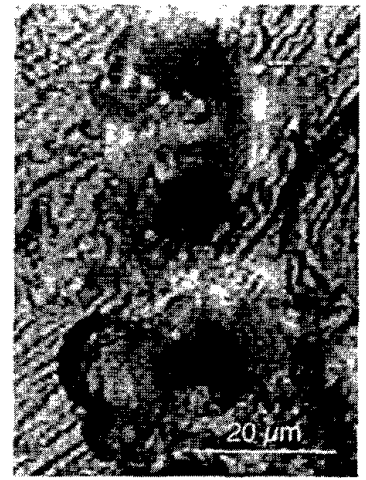

(a)

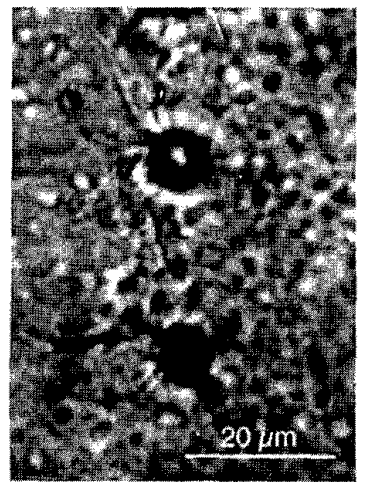

(c)

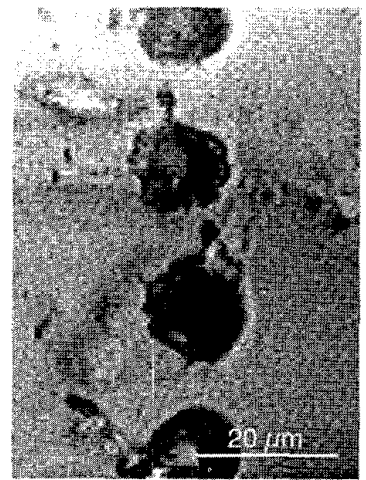

(b)

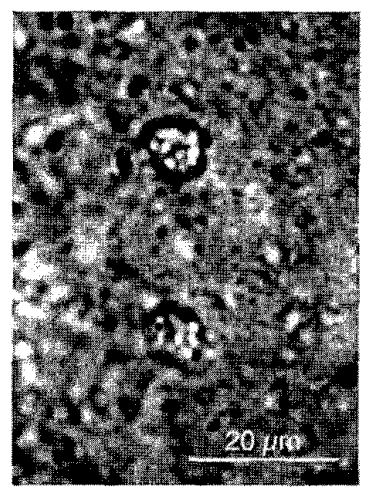

(d)
Fig. 2. (a) 200-ps, 40- $\mu \mathrm{J}$ ablation spot on skin surface. (b) 100-fs, $40-\mu \mathrm{J}$ ablation spot on skin surface. Both ablation regions have similar size, but the collateral tissue damage is much greater for the 200-ps pulses. (c) 200-ps, 20- $\mu \mathrm{J}$ ablation on surface. (d) 100-fs, 10$\mu \mathrm{J}$ ablation on surface. Again, the ablation regions for the two different pulse lengths are similar. However, the 200-ps pulse ablation region shows collateral tissue damage in a radiating pattern. All images are top-views of the surface.

clearly defined edges and the surrounding area is unaffected. The ablation threshold was determined as the lowest energy at which ablation spots were visible on the skin surface. For 100 -fs pulses the threshold was $\sim 2 \mu \mathrm{J}$ and the 200 -ps threshold was $\sim 5 \mu \mathrm{J}$. Figure $2 c$ and $2 d$ show surface ablation with a laser energy of five times the observed ablation threshold for each of the two different pulse lengths. Once again, the ablated regions have similar diameters of $\sim 3.5 \mu \mathrm{m}$. The edges of the 200-ps ablation spots are jagged and show radiating damage, while the 100 -fs ablation spots have smooth edges and show no collateral damage.

Figure 3 shows a series of cavities produced with $100-\mathrm{fs}$, $20-\mu \mathrm{J}$ pulses at varying depth beneath the surface. The cavities lie beneath the surface of the skin with no damage on the tissue surface itself. At $20 \mu \mathrm{J}$ of energy, 200-ps pulses caused

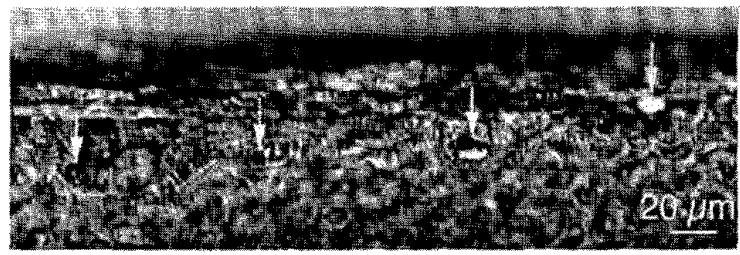

Fig. 3. Cross-sectional view of a series of cavities made in the bulk of skin with $100-\mathrm{fs}, 20-\mu \mathrm{J}$ pulses. Each pulse was focused at a different depth relative to the skin surface.

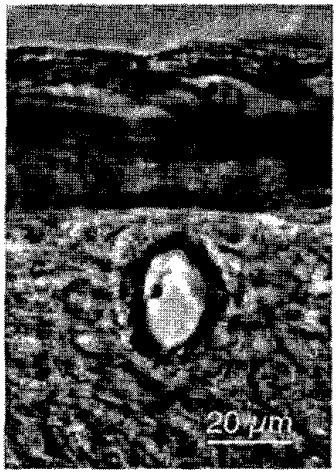

(a)

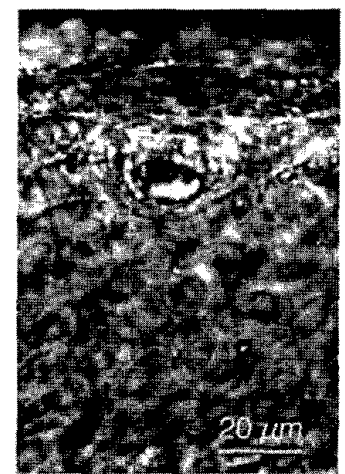

(b)
Fig. 4. (a) Sub-surface cavity created by $200-\mathrm{ps}, 20-\mu \mathrm{J}$ pulse. The cavity is surrounded by a thick ring of damaged tissue. (b) Subsurface cavity created by $100-\mathrm{fs}, 20-\mu \mathrm{J}$ pulse. This is a close-up of the third cavity from the left pictured in Fig. 3. Both images are cross-sectional views.

cavities which ruptured the back of the sample at a depth of about $\sim 100 \mu \mathrm{m}$. We did not see cavities from 100-fs pulses below about $\sim 50 \mu \mathrm{m}$ at $20 \mu \mathrm{J}$. Figure 4 shows cavities from 200 -ps and $100-\mathrm{fs}$ pulses at $20 \mu \mathrm{J}$. The cavities produced by 200 -ps pulses were larger and elongated along the axis of beam propagation, while cavities produced by 100 -fs pulses covered less than half the area of the picosecond cavities and were elongated perpendicular to the beam axis. In addition to having a larger size, cavities produced by picosecond pulses have more collateral tissue damage than those produced with 100 -fs pulses. The more extensive collateral damage produced by the picosecond pulses can be seen in Fig. $4 a$ as a thick dark ring around the irradiated area.

Figure 5 shows a region on the surface of the skin sample that has been ablated with multiple, $100-\mathrm{fs}, 4-\mu \mathrm{J}$ pulses. The sample was translated slowly under the laser beam, cutting a line across the skin surface. The width at the top of the ablated region is about $25 \mu \mathrm{m}$. The ablated region goes through the keratinized layer of the skin to a depth of about $25 \mu \mathrm{m}$. The edges of the ablation are straight and the surrounding tissue shows little damage. 


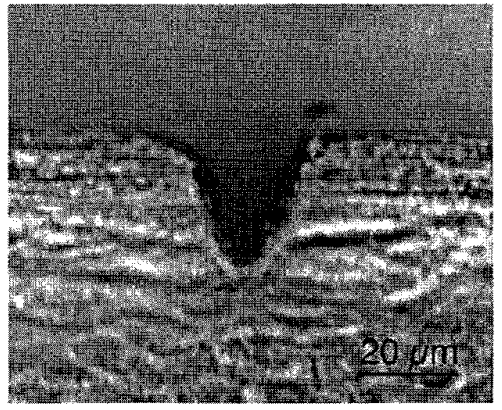

Fig. 5. Cross-section of $25 \mu$ m-deep incision produced by multiple-shot ablation with 100 -fs, $4-\mu \mathrm{J}$ laser pulses.

\section{Conclusions}

We investigated the potential of femtosecond and picosecond laser pulses for surgical use. We measured the breakdown threshold in water using a pressure wave detection technique. We find that femtosecond thresholds are significantly lower than picosecond thresholds. In addition, at energies near the breakdown threshold, picosecond pulses were unable to produce breakdown consistently. Femtosecond pulses, in contrast, produce breakdown with each pulse, even at threshold. We conclude that femtosecond pulses allow for very low energy deposition, while still consistently producing breakdown. Comparisons of laser-induced ablation with femtosecond and picosecond pulses in skin shows that the collateral tissue damage is much less extensive for shorter pulses. This is evident in both surface ablation and bulk cavity formation. On the surface, both femtosecond and picosecond pulses produce similar ablation spot sizes, but in the bulk, cavities were found to be smaller for femtosecond pulses. Finally, we demonstrated that femtosecond pulses can produce very clean, precise incisions with minimal collateral damage in tissue by multiple shot ablation. These findings show that femtosecond lasers offer potential advantages over picosecond lasers in surgical applications.

\section{Acknowledgments}

The authors thank Jill McMahon for help with the histology and the use of equipment. N. N. acknowledges the support of a Harvard Physics Department summer scholarship, and C. B. S. acknowledges the support of a National Defense Science and Engineering Fellowship.

\section{References}

1. Y. R. Shen, The Principles of Nonlinear Optics, New York: Wiley, 1984, pp. 528-540.

2. C. B. Schaffer, N. Nishimura, E. N. Glezer, and E. Mazur, "Ultrafast laser induced microexplosions: explosive dynamics and sub-micrometer structures," to appear in Proc. SPIE vol. 3269.

3. P. A. Barnes and K.E. Rieckoff, "Laser induced underwater sparks," Appl. Phys. Lett. vol. 13, pp. 282-284, 1968.

4. B. Zysset, J. G. Fujimoto, and T. F. Deutsch, "Time-resolved measurements of picosecond optical breakdown," Appl. Phys. B. vol. 48 , pp. 139-147, 1989.

5. T. Juhasz, G. A. Kastis, C. Suarez, Z. Bor, and W. E. Bron, "Time-resolved observations of shock waves and cavitation bubbles generated by femtosecond laser pulses in corneal tissue and water," Lasers Surg. Med. vol. 17, pp. 1-9, 1995.

6. B. Zysset, J. G. Fujimoto, C. A. Puliafito, R. Birngruber and T. F. Deutsch, "Picosecond optical breakdown: Tissue effects and reduction of collateral damage," Lasers Surg. Med. vol. 9, pp. 193-204, 1989.

7. J.M. Krauss and C. A. Puliafito, Lasers in Ophthalmology, in Laser Surgery and Medicine: Principals and Practice, ed. by C. A. Puliafito, Wiley-Liss, New York, 1996, Chap. 8.

8. R. F. Steinert and C. A. Puliafito, The Nd:YAG laser in Ophthalmology. Philadelphia, PA: Saunders, 1985

9. A. G. Doukas, A. D. Zweig, J. K. Frisoli, R. Bringruber, and T. F Deutsch, "Non-invasive determination of shock wave pressure generated by optical breakdown," Appl. Phys. B. vol. 53, pp. 237-245, 1991.

10. F. H. Loesel, J. P. Fischer, M. H. Gotz, C. Horvath, T. Juhs, F. Noack, N. Suhm, and J. F. Bille, "Non-thermal ablation of neural tissue with femtosecond laser pulses." Appl. Phys B. vol 66, pp. 121-128, 1998.

11. A. A. Oraevsky, L. B. DaSilva, A. M. Rubenchik, M. D. Feit, M. E. Glinsky, M. D. Perry, B. M. Mammini, W. S. Small IV, and B.C. Stuart, "Plasma mediated ablation of biological tissues with nanosecond-to-femtosecond laser pulses," IEEE J. Select. Topics Quantum Electron. vol.2, pp. 801-809, 1996.

12. J. Noack, D. X. Hammer, G. D. Noojin, B. A. Rockwell, and A. Vogel, "Influence of pulse duration on mechanical effects after laser-induced breakdown in water," J. Appl. Phys. vol. 83, pp. 7488-7495, 1998

13. E. N. Glezer, C. B. Schaffer, N. Nishimura, and E. Mazur, "Minimally distuptive laser-induced breakdown in water," Opt. Lett. vol. 22, pp. 1817-1819, 1997.

14. J. G. Fujimoto, W. Z. Lin, E. P. Ippen, C. A. Puliafito, and R. F. Steinert, "Time-resolved studies of Nd:YAG laser-induced breakdown," Invest. Ophthalmol. Vis. Sci. vol. 26, pp. 1771 $1777,1985$. 